\title{
Linear representations of subgeometries
}

\author{
Stefaan De Winter* Sara Rottey ${ }^{\dagger} \quad$ Geertrui Van de Voorde ${ }^{\ddagger}$
}

July 26, 2018

\begin{abstract}
The linear representation $T_{n}^{*}(\mathcal{K})$ of a point set $\mathcal{K}$ in a hyperplane of $\operatorname{PG}(n+1, q)$ is a point-line geometry embedded in this projective space. In this paper, we will determine the isomorphisms between two linear representations $T_{n}^{*}(\mathcal{K})$ and $T_{n}^{*}\left(\mathcal{K}^{\prime}\right)$, under a few conditions on $\mathcal{K}$ and $\mathcal{K}^{\prime}$. First, we prove that an isomorphism between $T_{n}^{*}(\mathcal{K})$ and $T_{n}^{*}\left(\mathcal{K}^{\prime}\right)$ is induced by an isomorphism between the two linear representations $T_{n}^{*}(\overline{\mathcal{K}})$ and $T_{n}^{*}\left(\overline{\mathcal{K}^{\prime}}\right)$ of their closures $\overline{\mathcal{K}}$ and $\overline{\mathcal{K}^{\prime}}$.

This allows us to focus on the automorphism group of a linear representation $T_{n}^{*}(\mathcal{S})$ of a subgeometry $\mathcal{S} \cong \mathrm{PG}(n, q)$ embedded in a hyperplane of the projective space $\mathrm{PG}\left(n+1, q^{t}\right)$. To this end we introduce a geometry $X(n, t, q)$ and determine its automorphism group. The geometry $X(n, t, q)$ is a straightforward generalization of $H_{q}^{n+2}$ which is known to be isomorphic to the linear representation of a Baer subgeometry. By providing an elegant algebraic description of $X(n, t, q)$ as a coset geometry we extend this result and prove that $X(n, t, q)$ and $T_{n}^{*}(\mathcal{S})$ are isomorphic.

Finally, we compare the full automorphism group of $T_{n}^{*}(\mathcal{S})$ with the "natural" group of automorphisms that is induced by the collineation group of its ambient space.
\end{abstract}

Keywords: Linear representation, automorphism group, subgeometry, coset geometry

MSC: $51 \mathrm{E} 20$

\section{Introduction}

In finite geometry, one often considers geometries that are embedded in a projective or affine space. If $G_{1}$ and $G_{2}$ are two such geometries, embedded in the same space, we may ask the following question:

\footnotetext{
*Supported by Michigan Technological University REF grant R01289

${ }^{\dagger}$ Partially supported by Michigan Technological University REF grant R01289

${ }^{\ddagger}$ Supported by the Fund for Scientific Research - Flanders (FWO).
} 
(Q) Is every isomorphism between $G_{1}$ and $G_{2}$ induced by a collineation of the ambient space?

Of course, the answer to this question will strongly depend on the properties of the geometries $G_{1}$ and $G_{2}$, as well as on the type of embedding considered. For example, it is well known that this question has an affirmative answer for the standard embeddings of finite classical polar spaces and Segre varieties [14, and has been studied for various other types of geometries such as generalised quadrangles [19] and semipartial geometries [9].

In this paper, we study the problem (Q) for a particular but broad class of geometries, namely linear representations.

The linear representation $T_{2}^{*}(\mathcal{O})$, where $\mathcal{O}$ is a hyperoval, was introduced by Ahrens and Szekeres [1] and independently by Hall [12]. This definition was extended to the linear representation of general point sets in [8].

Definition. Consider the projective space $\mathrm{PG}(n+1, q)$ for some prime power $q$. Let $H_{\infty}$ be a hyperplane in $\operatorname{PG}(n+1, q)$ and let $\mathcal{K}$ be a point set in $H_{\infty}$. The linear representation $T_{n}^{*}(\mathcal{K})$ of the point set $\mathcal{K}$ is the point-line incidence structure $(\mathcal{P}, \mathcal{L})$ whose points and lines are as follows:

$\mathcal{P}$ : the affine points of $\mathrm{PG}(n+1, q)$, i.e. the points of $\mathrm{PG}(n+1, q) \backslash H_{\infty}$,

$\mathcal{L}$ : the lines of $\operatorname{PG}(n+1, q)$ intersecting $H_{\infty}$ exactly in a point of $\mathcal{K}$.

Incidence is natural.

We see that a linear representation $T_{n}^{*}(\mathcal{K})$ in $\operatorname{PG}(n+1, q)$ is entirely determined by the point set $\mathcal{K}$ at infinity.

Linear representations are mostly studied for point sets $\mathcal{K}$ that possess a lot of symmetry. For example, in the case $n=2$ and $\mathcal{K}$ a hyperoval, $T_{2}^{*}(\mathcal{K})$ is a generalised quadrangle of order $(q-1, q+1)$. Bichara, Mazzocca and Somma showed in [4] that for $\mathcal{K}, \mathcal{K}^{\prime}$ hyperovals, $T_{2}^{*}(\mathcal{K}) \cong T_{2}^{*}\left(\mathcal{K}^{\prime}\right)$ if and only if $\mathcal{K}$ and $\mathcal{K}^{\prime}$ are PCL-equivalent. The answer to (Q) is given in the affirmative when $\mathcal{K}$ is a regular hyperoval in $\operatorname{PG}(2, q)$ in [11]. When $\mathcal{K}$ is a Buekenhout-Metz unital, question (Q) is answered by De Winter in [10; in this case, the linear representation $T_{2}^{*}(\mathcal{K})$ is a semipartial geometry. In [6] the authors proved that the answer to $(Q)$ for two linear representations $T_{n}^{*}(\mathcal{K})$ and $T_{n}^{*}\left(\mathcal{K}^{\prime}\right)$ is affirmative provided certain conditions on $\mathcal{K}$ are fulfilled (see Result 2.1). When one of these conditions is not met, the automorphism group can be larger. The same conditions might have been silently assumed when the authors of [2, Corollary 7] answer the question (Q) affirmatively in the case of (generalised) linear representations; since in [6] the authors have shown that the answer to question $(\mathrm{Q})$ is in general negative for linear representations. 
A frame of $\mathrm{PG}(n, q)$ is a set of $n+2$ points where each subset of $n+1$ points spans $\operatorname{PG}(n, q)$. When a point set $\mathcal{K}$ of $\operatorname{PG}(n, q)$ contains a frame, the closure $\overline{\mathcal{K}}$ of $\mathcal{K}$ consists of the points of the smallest subgeometry of $\operatorname{PG}(n, q)$ containing $\mathcal{K}$. The case $\overline{\mathcal{K}}=H_{\infty}$ was treated in [6]. This article is concerned with finding the full automorphism group of $T_{n}^{*}(\mathcal{K})$ in the case $\overline{\mathcal{K}} \neq H_{\infty}$

In Section 2 we prove that an isomorphism between $T_{n}^{*}(\mathcal{K})$ and $T_{n}^{*}\left(\mathcal{K}^{\prime}\right)$ is induced by an isomorphism between $T_{n}^{*}(\overline{\mathcal{K}})$ and $T_{n}^{*}\left(\overline{\mathcal{K}^{\prime}}\right)$. This explains why the rest of the paper is devoted to isomorphisms between $T_{n}^{*}(\mathcal{S})$ and $T_{n}^{*}\left(\mathcal{S}^{\prime}\right)$ for subgeometries $\mathcal{S} \cong \mathcal{S}^{\prime}$ of $H_{\infty}$.

In Section 3 we introduce the geometry $X(n, t, q)$, which is a generalisation of the semipartial geometry $H_{q}^{n+2}$ that was introduced in [8]. We will explore the automorphism group of $X(n, t, q)$ and prove that this group consists solely of collineations of its ambient space $\mathrm{PG}(n+t+1, q)$.

In Section 4 we study $X(n, t, q)$ as a coset geometry, generalising the idea of translation semipartial geometries as introduced in [9]. In a group theoretical / algebraic way, we recover the isomorphism with $T_{n}^{*}(\mathcal{S})$, where $\mathcal{S}$ is a subgeometry $\mathrm{PG}(n, q)$ of the hyperplane $H_{\infty} \cong \mathrm{PG}\left(n, q^{t}\right)$. This yields a useful and elegant algebraic description of $T_{n}^{*}(\mathcal{S})$ and its full automorphism group. It, for example, allows an explicit description of the automorphism group making it possible to perform computations in this group.

In Section 5 we prove that the automorphism group of $T_{n}^{*}(\mathcal{S})$ is isomorphic to a specific collineation group of $\mathrm{PG}(t(n+1)+1, q)$, namely the collineation group stabilising the generalised linear representation isomorphic to $T_{n}^{*}(\mathcal{S})$. These results in particular show that the automorphism group of $T_{n}^{*}(\mathcal{S})$ is much larger than its automorphism group induced by $\operatorname{P\Gamma L}(n+2, q)$. We end with a full answer to the isomorphism problem of linear representations $T_{n}^{*}(\mathcal{K})$ and $T_{n}^{*}\left(\mathcal{K}^{\prime}\right)$ of point sets $\mathcal{K}, \mathcal{K}^{\prime}$ in $\operatorname{PG}\left(n, q^{t}\right)$, such that the closure of $\mathcal{K}, \mathcal{K}^{\prime}$ is a subgeometry $\mathrm{PG}(n, q)$.

\section{Linear representations}

First of all, we wish to emphasize the distinction between a subspace and a subgeometry. A subspace of $\mathrm{PG}(n, q)$ is a projective space $\mathrm{PG}(m, q)$ contained in $\mathrm{PG}(n, q), m \leq n$, over the same finite field $\mathbb{F}_{q}$. Now consider a subfield $\mathbb{F}_{q_{0}}$ of $\mathbb{F}_{q}$; an $n$-dimensional subgeometry of $\operatorname{PG}(n, q)$ of order $q_{0}$ is a set of $\left(q_{0}^{n+1}-1\right) /\left(q_{0}-1\right)$ points whose homogeneous coordinates, with respect to a fixed frame of $\mathrm{PG}(n, q)$, are in $\mathbb{F}_{q_{0}}$.

In this paper we will consider a linear representation $T_{n}^{*}(\mathcal{K})$ of a point set $\mathcal{K}$ contained in a hyperplane $H_{\infty}$, containing a frame and satisfying specific conditions. In order to state these conditions we need the following two definitions.

Definition. We say a point set $\mathcal{K}$ of $\operatorname{PG}(n, q)$ has Property (*) if there is 
no plane of $\operatorname{PG}(n, q)$ intersecting $\mathcal{K}$ in only two intersecting lines or in two intersecting lines minus their intersection point.

We will exclude all point sets that do not possess Property (*). Note that in [6], considering a linear representation of a specific point set not having this property, an explicit example of an automorphism that is not a collineation of the ambient space is given.

Definition. If a point set $S$ of $\mathrm{PG}(n, q)$ contains a frame of $\mathrm{PG}(n, q)$, then its closure $\bar{S}$ consists of the points of the smallest $n$-dimensional subgeometry of $\mathrm{PG}(n, q)$ containing all points of $S$.

The closure $\bar{S}$ of a point set $S$ can be constructed recursively as follows:

(i) determine the set $\mathcal{A}$ of all subspaces of $\mathrm{PG}(n, q)$ spanned by an arbitrary number of points of $S$;

(ii) determine the set $\bar{S}$ of all points that arise as the exact intersection of any two subspaces in $\mathcal{A}$, that is, $P \in \bar{S}$ if and only there exist two subspaces $\pi_{1}$ and $\pi_{2}$ spanned by points of $S$, such that $P=\pi_{1} \cap \pi_{2}$. If $\bar{S} \neq S$ replace $S$ by $\bar{S}$ and go to (i), otherwise stop.

For $n=2$, this recursive construction coincides with the definition of the closure of a set of points in a plane containing a quadrangle, given in [16, Chapter XI].

The result in [6] concerning the isomorphisms between linear representations $T_{n}^{*}(\mathcal{K})$ and $T_{n}^{*}\left(\mathcal{K}^{\prime}\right)$ for point sets $\mathcal{K}$ and $\mathcal{K}^{\prime}$, is the following.

Result 2.1. 6] Let $q>2$. Let $\mathcal{K}$ and $\mathcal{K}^{\prime}$ denote point sets spanning $H_{\infty} \cong$ $\mathrm{PG}(n, q)$, having Property (*) in $H_{\infty}$, such that the closure $\overline{\mathcal{K}}$ is equal to $H_{\infty}$. Let $\alpha$ be an isomorphism between $T_{n}^{*}(\mathcal{K})$ and $T_{n}^{*}\left(\mathcal{K}^{\prime}\right)$. Then $\alpha$ is induced by an element of the stabiliser $\operatorname{P\Gamma L}(n+2, q)_{H_{\infty}}$ of $H_{\infty}$ mapping $\mathcal{K}$ to $\mathcal{K}^{\prime}$.

We will prove that, if $\mathcal{K}$ or $\mathcal{K}^{\prime}$ satisfies Property (*), an isomorphism between the linear representations $T_{n}^{*}(\mathcal{K})$ and $T_{n}^{*}\left(\mathcal{K}^{\prime}\right)$ of two point sets $\mathcal{K}$ and $\mathcal{K}^{\prime}$ is always induced by an isomorphism between the linear representations $T_{n}^{*}(\overline{\mathcal{K}})$ and $T_{n}^{*}\left(\overline{\mathcal{K}^{\prime}}\right)$ of their closures $\overline{\mathcal{K}}$ and $\overline{\mathcal{K}^{\prime}}$.

We need the notion of $\alpha$-rigid subspaces (see [6]):

Definition. Let $\alpha$ be an isomorphism between $T_{n}^{*}(\mathcal{K})$ and $T_{n}^{*}\left(\mathcal{K}^{\prime}\right)$. We will say that a $k$-subspace $\pi_{\infty}$ of $H_{\infty}$ is $\alpha$-rigid if for every $(k+1)$-subspace $\pi$ containing $\pi_{\infty}$, but not contained in $H_{\infty}$, the point set $\{\alpha(P) \mid P \in \pi, P \notin$ $\left.H_{\infty}\right\}$ spans a $(k+1)$-subspace.

It follows from the definition of a linear representation $T_{n}^{*}(\mathcal{K})$ that every point of $\mathcal{K}$ is $\beta$-rigid, for any isomorphism $\beta$.

We will need the following theorems of [6]. 
Result 2.2. [6] If for some isomorphism $\alpha$, the subspaces $\pi_{1}$ and $\pi_{2}$ are $\alpha$-rigid subspaces meeting in at least one point, then $\pi_{1} \cap \pi_{2}$ is an $\alpha$-rigid subspace.

For a line $L$ of $\operatorname{PG}(n+1, q)$ not in $H_{\infty}$, we define $\infty(L)$ to be the point $L \cap H_{\infty}$.

An isomorphism $\beta$ of the affine points extends naturally to a mapping on the lines $L$ having a $\beta$-rigid point $\infty(L)$ at infinity, by defining $\beta(L)$ to be the line containing the points $\beta(R), R \in L$.

Result 2.3. 6] Let $q>2$. Suppose $\mathcal{K}$ or $\mathcal{K}^{\prime}$ has Property $(*)$ and $\langle\mathcal{K}\rangle=$ $\left\langle\mathcal{K}^{\prime}\right\rangle=H_{\infty}$. Let $\alpha$ be an isomorphism between $T_{n}^{*}(\mathcal{K})$ and $T_{n}^{*}\left(\mathcal{K}^{\prime}\right)$. We can define a mapping $\tilde{\alpha}$ on the set of $\alpha$-rigid points by putting $\tilde{\alpha}(Q)=\infty(\alpha(L))$ where $Q$ is an $\alpha$-rigid point and $L$ is any line for which $\infty(L)=Q$. This means, for two lines $L$ and $M$, if $\infty(L)=\infty(M)=Q$ is an $\alpha$-rigid point, then $\infty(\alpha(L))=\infty(\alpha(M))=\tilde{\alpha}(Q)$.

This result shows that an isomorphism $\beta$ between $T_{n}^{*}(\mathcal{K})$ and $T_{n}^{*}\left(\mathcal{K}^{\prime}\right)$ can be extended to a mapping on the $\beta$-rigid points $Q \in H_{\infty}$, and we abuse notation by putting $\beta(Q):=\tilde{\beta}(Q)$.

Result 2.4. 6] Let $q>2$. Suppose $\mathcal{K}$ or $\mathcal{K}^{\prime}$ has Property $(*)$ and $\langle\mathcal{K}\rangle=$ $\left\langle\mathcal{K}^{\prime}\right\rangle=H_{\infty}$. Let $\alpha$ be an isomorphism between $T_{n}^{*}(\mathcal{K})$ and $T_{n}^{*}\left(\mathcal{K}^{\prime}\right)$. If $P_{1}, \ldots, P_{k+1}$ are $\alpha$-rigid points, then $\left\langle P_{1}, \ldots, P_{k+1}\right\rangle$ is an $\alpha$-rigid space.

In the following theorem we generalise Result 2.1 for point sets $\mathcal{K}$ whose closure is not necessarily $H_{\infty}$.

Theorem 2.5. Let $\mathcal{K}$ and $\mathcal{K}^{\prime}$ be point sets in the hyperplane $H_{\infty} \cong \mathrm{PG}(n, q)$ such that $\mathcal{S}=\overline{\mathcal{K}}$ and $\mathcal{S}^{\prime}=\overline{\mathcal{K}^{\prime}}$ are $n$-dimensional subgeometries of $H_{\infty}$. If $\mathcal{S}=H_{\infty}$, then further suppose $\mathcal{K}$ satisfies Property $(*)$. An isomorphism $\gamma$ between $T_{n}^{*}(\mathcal{K})$ and $T_{n}^{*}\left(\mathcal{K}^{\prime}\right)$ is induced by an isomorphism between $T_{n}^{*}(\mathcal{S})$ and $T_{n}^{*}\left(\mathcal{S}^{\prime}\right)$ that maps $\mathcal{S}$ onto $\mathcal{S}^{\prime}$.

Proof. If the subgeometry $\mathcal{S}$ is not the whole hyperplane $H_{\infty}$, then the set $\mathcal{K}$ spans $H_{\infty}$, but it does not contain full lines of $H_{\infty}$, nor full lines minus one point, and thus it also has Property $(*)$. By the recursive construction of the closure of a set of points, we conclude, invoking Result 2.2 and Result 2.4. that all points of $\mathcal{S}$ are $\gamma$-rigid. Hence $\gamma$ maps the affine points of a projective line intersecting $H_{\infty}$ in exactly one point of $\mathcal{S}$, onto the affine points of a projective line intersecting $H_{\infty}$ in exactly one point.

Making use of Result 2.3, we know that lines through a $\gamma$-rigid point at infinity are mapped to lines intersecting each other in a point at infinity. As said before, we abuse notation and use $\gamma$ for the extension of $\gamma$ to all $\gamma$-rigid points of $H_{\infty}$. Now, let $P, Q, R$ be three points of $\mathcal{S}$ on a line $L$ of $H_{\infty}$ and let $U$ be a point, not contained in $H_{\infty}$. Since $L$ is a $\gamma$-rigid line, 
$\gamma$ maps the points of $\langle U, L\rangle$ onto points of a plane containing $\gamma(P), \gamma(Q)$, and $\gamma(R)$ at infinity. This implies that $\gamma$ also maps collinear points of $\mathcal{S}$ to collinear points of $H_{\infty}$. Since $\mathcal{K}$ is mapped to $\mathcal{K}^{\prime}$, and collinearity needs to be preserved, clearly the points of $\mathcal{S}$ are mapped to the points of $\mathcal{S}^{\prime}$ (keeping the recursive construction of $\mathcal{S}$ and $\mathcal{S}^{\prime}$ in mind).

With the same argument, the points of $\mathcal{S}^{\prime}$ are $\gamma^{-1}$-rigid, and collinear points of $\mathcal{S}^{\prime}$ are mapped by $\gamma^{-1}$ to collinear points of $H_{\infty}$, thus belonging to $\mathcal{S}$. We conclude that $\gamma$ is induced by an isomorphism between $T_{n}^{*}(\mathcal{S})$ and $T_{n}^{*}\left(\mathcal{S}^{\prime}\right)$ that maps $\mathcal{S}$ onto $\mathcal{S}^{\prime}$ (preserving collinearity of points of $\mathcal{S}$ ).

The automorphism group of $T_{n}^{*}(\mathcal{K})$ where $\overline{\mathcal{K}}=H_{\infty}$ has been determined in [6]. It follows from Theorem 2.5 that we only need to consider the automorphism group of $T_{n}^{*}(\mathcal{S})$ where $\mathcal{S}$ is a subgeometry of $H_{\infty}$.

\section{The geometry $X(n, t, q)$ and its automorphism group}

Definition. Consider an $n$-dimensional subspace $\pi$ of the projective space $\operatorname{PG}(n+t, q)$. The geometry $X(n, t, q)$ is the incidence structure $(\mathcal{P}, \mathcal{L})$ with natural incidence and with

$\mathcal{P}$ : the $(t-1)$-spaces of $\mathrm{PG}(n+t, q)$ skew to $\pi$,

$\mathcal{L}$ : the $t$-spaces of $\mathrm{PG}(n+t, q)$ meeting $\pi$ in exactly one point.

For $t=2$, this geometry was introduced in [8] as $H_{q}^{n+2}$. The geometry $H_{q}^{n+2}$ was also given in [7] as an example of a semipartial geometry that is not a partial geometry for $n \geq 2$. There the author proved that for $n=2$ the geometry $H_{q}^{4}$ is isomorphic to $T_{2}^{*}(\mathcal{B})$ where $\mathcal{B}$ is a Baer subplane of $\mathrm{PG}\left(2, q^{2}\right)$. In Section 4 we will prove this for general $n$ and $t$, namely $X(n, t, q) \cong T_{n}^{*}(\mathcal{S})$, where $\mathcal{S}$ is an $n$-dimensional subgeometry over $\mathbb{F}_{q}$ of $\mathrm{PG}\left(n, q^{t}\right)$.

First, we will determine the full automorphism group of $X(n, t, q)$.

Consider the embedding of the geometry $X(n, t, q)$ in the projective space $\mathrm{PG}(n+t, q)$ where $\pi$ is an $n$-dimensional subspace. We will prove that an automorphism of $X(n, t, q)$ is induced by a collineation of its ambient space.

Lemma 3.1. All automorphisms of $X(n, t, q)$ are induced by collineations of its ambient space; more precisely, $\operatorname{Aut}(X(n, t, q)) \cong \mathrm{P} \Gamma \mathrm{L}(n+t+1, q)_{\pi}$.

Proof. We will prove that every automorphism of $X(n, t, q)$ is induced by a mapping on the points and lines of $\mathrm{PG}(n+t, q)$ which is incidence preserving, hence is a collineation.

Every automorphism $\psi$ of $X(n, t, q)$ is a permutation of the $(t-1)$ dimensional subspaces of $\mathrm{PG}(n+t, q)$ disjoint from $\pi$. Consider a $(t-2)$ dimensional space $\mu$ disjoint from $\pi$, and take two elements $\nu_{1}, \nu_{2} \in \mathcal{P}$ such 
that $\nu_{1} \cap \nu_{2}=\mu$. We clearly have $\left\langle\nu_{1}, \nu_{2}\right\rangle \in \mathcal{L}$. Since $\psi$ preserves $\mathcal{L}, \psi$ sends $\nu_{1}$ and $\nu_{2}$ to two elements $\nu_{1}^{\prime}$ and $\nu_{2}^{\prime}$ of $\mathcal{P}$ lying in an element of $\mathcal{L}$, thus intersecting in a $(t-2)$-dimensional space $\mu^{\prime}$.

Now consider $\nu_{3} \in \mathcal{P}$ containing $\mu$, but not lying in $\left\langle\nu_{1}, \nu_{2}\right\rangle$. As seen before, its image $\nu_{3}^{\prime}$ intersects both $\nu_{1}^{\prime}$ and $\nu_{2}^{\prime}$ in a $(t-2)$-dimensional space. Hence $\nu_{3}^{\prime} \cap \nu_{1}^{\prime}=\nu_{3}^{\prime} \cap \nu_{2}^{\prime}=\nu_{1}^{\prime} \cap \nu_{2}^{\prime}=\mu^{\prime}$, since otherwise $\nu_{1}^{\prime}, \nu_{2}^{\prime}$ and $\nu_{3}^{\prime}$ would lie in one and the same element of $\mathcal{L}$, a contradiction. It follows that $\psi$ extends to a well-defined mapping on the $(t-2)$-dimensional subspaces $\mu$ of $\operatorname{PG}(n+t, q)$ disjoint from $\pi$ by putting $\psi(\mu):=\psi\left(\nu_{1}\right) \cap \psi\left(\nu_{2}\right)$ for $\nu_{1}, \nu_{2} \in \mathcal{P}$ with $\nu_{1} \cap \nu_{2}=\mu$. Furthermore, $\psi$ preserves incidence between these $(t-2)$ spaces and the $(t-1)$-spaces of $\mathcal{P}$.

In other words, for $k=t-1$ the map $\psi$ extends to a mapping:

(i) permuting $(k+1)$-subspaces intersecting $\pi$ in exactly one point, such that incidence with the $k$-subspaces disjoint from $\pi$ is preserved,

(ii) permuting $k$-subspaces disjoint from $\pi$,

(iii) permuting $(k-1)$-subspaces disjoint from $\pi$, such that incidence with the $k$-subspaces disjoint from $\pi$ is preserved and such that incidence with the $(k+1)$-subspaces intersecting $\pi$ in one point is preserved.

Now we continue by induction. Suppose that for some $k \leq t-1$, the previous three properties are valid. We will prove they also hold for $k-1$. Clearly, property (ii) for $k-1$ follows from property (iii) for $k$. We proceed in two steps, first proving (i) and afterwards (iii).

Consider a $k$-dimensional space $\alpha$ intersecting $\pi$ in exactly one point $P$, and take two $(k+1)$-dimensional spaces $\beta_{1}, \beta_{2}$ intersecting $\pi$ only in $P$ such that $\beta_{1} \cap \beta_{2}=\alpha$. The map $\psi$ preserves the $(k-1)$-dimensional spaces of $\mathrm{PG}(n+t, q)$, disjoint from $\pi$, contained in $\alpha$ and their incidence with $\beta_{1}$ and $\beta_{2}$. The subspaces $\beta_{1}$ and $\beta_{2}$ are mapped to two $(k+1)$-dimensional spaces $\beta_{1}^{\prime}$ and $\beta_{2}^{\prime}$, intersecting in a subspace $\alpha^{\prime}$. The subspace $\alpha^{\prime}$ needs to contain exactly $q^{k}(k-1)$-dimensional spaces of $\mathrm{PG}(n+t, q)$ disjoint from $\pi$, hence $\alpha^{\prime}$ is a $k$-dimensional subspace intersecting $\pi$ in a point $P^{\prime}$. It follows that this point $P^{\prime}$ is the point at infinity for both $\beta_{1}^{\prime}$ and $\beta_{2}^{\prime}$.

Consider a third $(k+1)$-dimensional space $\beta_{3}$, intersecting $\pi$ in exactly $P$, and containing $\alpha$. Its image $\beta_{3}^{\prime}$, intersects both $\beta_{1}^{\prime}$ and $\beta_{2}^{\prime}$ in a $k$-dimensional space. Since $\psi$ acts bijectively on $(k-1)$-dimensional subspaces disjoint from $\pi$ and preserves their incidence with $(k+1)$-spaces intersecting $\pi$ in one point, the intersections $\beta_{3}^{\prime} \cap \beta_{1}^{\prime}, \beta_{3}^{\prime} \cap \beta_{2}^{\prime}$ and $\beta_{1}^{\prime} \cap \beta_{2}^{\prime}$ all contain the same $(k-1)$-dimensional subspaces and, again by counting, we see that they each coincide with the same $k$-dimensional subspace $\alpha^{\prime}$ intersecting $\pi$ in $P^{\prime}$. It follows that $\psi$ extends to a well-defined mapping on the $k$-dimensional subspaces of $\mathrm{PG}(n+t, q)$ intersecting $\pi$ in one point, preserving incidence with the $(k-1)$-dimensional subspaces disjoint from $\pi$. 
We now continue with the second step of the induction. Consider a $(k-2)$-dimensional subspace $\gamma$ of $\mathrm{PG}(n+t, q)$ disjoint from $\pi$. Take two $(k-1)$-dimensional subspaces $\delta_{1}$ and $\delta_{2}$, disjoint from $\pi$, such that $\delta_{1} \cap \delta_{2}=\gamma$ and such that $\left\langle\delta_{1}, \delta_{2}\right\rangle=\epsilon$ is a $k$-dimensional space intersecting $\pi$ in exactly one point $Q$. From the previous part, we have that their images $\delta_{1}^{\prime}$ and $\delta_{2}^{\prime}$ are contained in $\epsilon^{\prime}$, which is the image of $\epsilon$, hence they intersect in a $(k-2)$-dimensional subspace $\gamma^{\prime}$, disjoint from $\pi$.

Consider a third $(k-1)$-dimensional subspace $\delta_{3}$ containing $\gamma$, disjoint from $\pi$, not contained in $\epsilon$, such that $\left\langle\delta_{1}^{\prime}, \delta_{2}^{\prime}, \delta_{3}^{\prime}\right\rangle$ is a $(k+1)$-dimensional space intersecting $\pi$ in the point $Q$. From the above, the image $\delta_{3}^{\prime}$ intersects both $\delta_{1}^{\prime}$ and $\delta_{2}^{\prime}$ in a $(k-2)$-dimensional space. The intersection $\delta_{3}^{\prime} \cap \delta_{1}^{\prime}$ equals $\delta_{3}^{\prime} \cap \delta_{2}^{\prime}$, since otherwise $\left\langle\delta_{1}, \delta_{2}, \delta_{3}\right\rangle$ would be $k$-dimensional, a contradiction because $\psi$ preserves $(k+1)$-dimensional spaces intersecting $\pi$ in exactly in one point.

Hence, the map $\psi$ extends to a well-defined mapping on the $(k-2)$ dimensional subspaces of $\mathrm{PG}(n+t, q)$, disjoint from $\pi$, preserving incidence with the $k$-dimensional subspaces intersecting $\pi$ in one point, and with the $(k-1)$-dimensional subspaces disjoint from $\pi$.

By induction we see that the map $\psi$ extends to a well-defined incidence preserving mapping on the $m$-dimensional spaces, $0 \leq m \leq t-1$, disjoint from $\pi$ and the $l$-dimensional spaces, $1 \leq l \leq t$, intersecting $\pi$ in exactly one point.

We now only need to show that $\psi$ can be extended to a bijective mapping on the points of $\pi$. Suppose that $P$ is a point of $\pi$, and $L_{1}$ and $L_{2}$ are two lines such that $L_{1} \cap \pi=L_{2} \cap \pi=\{P\}$. Since the plane $\left\langle L_{1}, L_{2}\right\rangle$ is mapped to a plane intersecting $\pi$ in one point $P^{\prime}$, both $L_{1}$ and $L_{2}$ are mapped to lines intersecting $\pi$ in $P^{\prime}$. Consider a line $L_{3}$ intersecting $\pi$ in exactly $P$, such that $\left\langle L_{1}, L_{3}\right\rangle$ intersects $\pi$ in a line. Then $\left\langle L_{2}, L_{3}\right\rangle$ intersects $\pi$ only in $P$, hence also $L_{3}$ is mapped to a line intersecting $\pi$ in $P^{\prime}$. So we can extend $\psi$ to a mapping on the points of $\pi$ by putting $\psi(P):=\psi\left(L_{1}\right) \cap \psi\left(L_{2}\right)$ for $L_{1}, L_{2}$ lines meeting $\pi$ exactly in the point $P$.

Now consider a line $L$ in $\pi$. Take a 3 -dimensional space $\mu$ intersecting $\pi$ in exactly $L$. Take two disjoint lines $M \neq L$ and $N \neq L$ in $\mu$; they are mapped to two disjoint lines spanning a 3 -dimensional space $\mu^{\prime}$ intersecting $\pi$ in at most a line. The $q+1$ planes, spanned by $M$ and a point of $L$, all intersect $N$ in a point, hence their images lie in $\mu^{\prime}$. The images of the points of $L$ are all different and lie in $\mu^{\prime}$, hence they lie on a line $L^{\prime}$ which has to be the intersection of $\mu^{\prime}$ with $\pi$. It follows that $\psi$ also preserves lines of $\pi$.

We proved that every automorphism $\psi$ of $X(n, t, q)$ is induced by a mapping on the points and lines of $\mathrm{PG}(n+t, q)$ which is incidence preserving, hence is a collineation. From this, it is clear that the automorphism group of $X(n, t, q)$ is isomorphic to the stabiliser $\operatorname{P\Gamma L}(n+t+1, q)_{\pi}$ of $\pi$. 


\section{$4 X(n, t, q)$ as a coset geometry}

In this section we will see that $X(n, t, q)$ has a natural description as a coset geometry. We will prove that $X(n, t, q)$ is isomorphic to the linear representation $T_{n}^{*}(\mathcal{S})$ embedded in $\mathrm{PG}\left(n+1, q^{t}\right)$, where $\mathcal{S}$ is the subgeometry $\mathrm{PG}(n, q)$ of the hyperplane $H_{\infty} \cong \mathrm{PG}\left(n, q^{t}\right)$. Moreover, this will provide an elegant description of both the geometry and its automorphism group as determined in Section 3 .

Without loss of generality, let $\pi$ be the $n$-dimensional space of $\mathrm{PG}(n+t, q)$ with equation $X_{0}=X_{1}=\ldots=X_{t-1}=0$. We consider the embedding of the geometry $X(n, t, q)$ in $\mathrm{PG}(n+t, q)$. Recall that the point set $\mathcal{P}$ consists of the $(t-1)$-subspaces of $\mathrm{PG}(n+t, q)$ disjoint from $\pi$, and the line set $\mathcal{L}$ consists of the $t$-subspaces of $\mathrm{PG}(n+t, q)$ intersecting $\pi$ in exactly one point.

Consider the following $(n+1)$-dimensional spaces through $\pi: \Sigma_{0}: X_{1}=$ $\ldots=X_{t-1}=0, \Sigma_{j}: X_{0}=\ldots=X_{j-1}=X_{j+1}=\ldots=X_{t-1}=0, j=$ $1, \ldots, t-2, \Sigma_{t-1}: X_{0}=\ldots=X_{t-2}=0$. Every $(t-1)$-dimensional space $P \in$ $\mathcal{P}$ is spanned by the set of $t$ unique points $\left\{U_{j}=P \cap \Sigma_{j}\right\}_{j=0, \ldots, t-1}$, where $U_{j}$ has coordinates $\left(0, \ldots, 0,1,0, \ldots, 0, a_{0 j}, \ldots, a_{n j}\right)$ with a 1 at position $j$ and $a_{i j} \in \mathbb{F}_{q}$, for all $i=0, \ldots, n$, and $j=0, \ldots, t-1$.

For every $P \in \mathcal{P}$ we can consider a corresponding $(n+t+1) \times(n+t+1)$ matrix $A_{P}^{\prime}$

$$
A_{P}^{\prime}=\left(\begin{array}{cc}
I_{t} & 0 \\
A_{P} & I_{n+1}
\end{array}\right)
$$

with the $(n+1) \times t$-matrix $A_{P}=\left(a_{i j}\right), 0 \leq i \leq n, 0 \leq j \leq t-1$, and where $I_{k}$ is the $k \times k$-identity matrix. Conversely every such matrix corresponds to a unique $P \in \mathcal{P}$.

Now let $G$ be the set consisting of all these matrices,

$$
G=\left\{A_{P}^{\prime} \mid P \in \mathcal{P}\right\} .
$$

This set $G$, under operation of multiplication, forms a group. Note that

$$
A_{P}^{\prime} A_{Q}^{\prime}=\left(\begin{array}{cc}
I_{t} & 0 \\
A_{P}+A_{Q} & I_{n+1}
\end{array}\right)
$$

Hence, this group is elementary abelian of order $q^{(n+1) t}$. Furthermore, since every elementary abelian group corresponds to a vector space, this implies we will be able to interpret $X(n, t, q)$ as a geometry "embedded" in an $\mathbb{F}_{p^{-}}$ vector space, or equivalently, in an affine space over $\mathbb{F}_{p}$.

We define the action of $G$ on $\mathcal{P}$ by $A_{R}^{\prime}(P)=Q$ if and only if $A_{R}^{\prime} A_{P}^{\prime}=A_{Q}^{\prime}$. It is obvious that this is well defined and that the group $G$ acts sharply transitively on the point set $\mathcal{P}$ of $X(n, t, q)$.

One could also envision $G$ as a subgroup of $\operatorname{PGL}(n+t+1, q)$ and consider the action on the points of $\mathrm{PG}(n+t, q)$ by left-multiplication. The induced 
action on the elements of $\mathcal{P}$ is exactly the same as the one we defined earlier. Note that all the points of $\pi$ are fixed by the group $G$.

Next we will describe the lines of $X(n, t, q)$ in an algebraic way. Consider for every point $V \in \pi$ its coordinates $\left(0, \ldots, 0, b_{0}, \ldots, b_{n}\right)$ (determined up to scalar multiple). We define the subgroup $G(V)$ of $G$ as follows:

$$
G(V)=\left\{\left(\begin{array}{cc}
I_{t} & 0 \\
B_{\bar{a}} & I_{n+1}
\end{array}\right) \mid \bar{a} \in \mathbb{F}_{q}^{t}\right\}
$$

with the $(n+1) \times t$-matrix $B_{\bar{a}}=\left(b_{i} a_{j}\right), 0 \leq i \leq n, 0 \leq j \leq t-1$ for $\bar{a}=\left(a_{0}, a_{1}, \ldots, a_{t-1}\right) \in \mathbb{F}_{q}^{t}$. Clearly the group $G(V)$ is independent of the chosen coordinates for $V$.

For $0 \leq i \leq n+t$, suppose the point $W_{i}$ of $\mathrm{PG}(n+t, q)$ has coordinates $w_{i}$, where $w_{0}=(1,0, \ldots, 0), w_{1}=(0,1,0, \ldots, 0), \ldots, w_{n+t}=(0, \ldots, 0,1)$. The group $G(V)$ has size $q^{t}$ and stabilises the $t$-space $L_{V}=\left\langle V, W_{0}, W_{1}, \ldots, W_{t-1}\right\rangle$, note that this space is an element of $\mathcal{L}$. The group $G(V)$ fixes the point $V$ and acts transitively on the $t$-tuples $\left(V_{0}, \ldots, V_{t-1}\right)$, where $V_{i}$ is a point of $\left\langle V, W_{i}\right\rangle \backslash\{V\}$. Hence, it acts transitively on the $(t-1)$-spaces of $L_{V}$ not through $V$. These are exactly the elements of $\mathcal{P}$ contained in this line $L_{V}$ of $X(n, t, q)$. Furthermore, since this group has size $q^{t}$, this action is sharply transitive.

The space $I=\left\langle W_{0}, \ldots, W_{t-1}\right\rangle \in \mathcal{P}$ corresponds to the identity matrix of $G$. From the above, we learned that the lines of $X(n, t, q)$ through $I$ correspond to the subgroups $G(V), V \in \pi$. Furthermore, since $G$ (interpreted as a subgroup of $\operatorname{PGL}(n+t+1, q))$ fixes all points of $\pi$ and acts (sharply) transitively on the $(t-1)$-spaces disjoint from $\pi$, we easily deduce that the elements of $\mathcal{L}$ (the lines of $X(n, t, q)$ ) are in one to one correspondence with the cosets of $G(V)$ in $G$. However, at this point we can simplify notation if we take into account that all important properties of $G$ and the subgroups $G(V)$ are determined by the $(n+1) \times t$-submatrices in the lower left corner of the elements of $G$. Let $\mathbf{M}=M_{q}(n+1, t)$ be the group of all $(n+1) \times t$ matrices over $\mathbb{F}_{q}$ under matrix addition. We have obtained the following description of $X(n, t, q)$ as a coset geometry $\mathcal{M}=\left(\mathcal{P}_{\mathcal{M}}, \mathcal{L}_{\mathcal{M}}\right)$ with natural incidence (containment), point set $\mathcal{P}_{\mathcal{M}}$ and line set $\mathcal{L}_{\mathcal{M}}$ as follows:

$\mathcal{P}_{\mathcal{M}}$ : the elements of $\mathbf{M}$, that is, the $(n+1) \times t$-matrices over $\mathbb{F}_{q}$,

$\mathcal{L}_{\mathcal{M}}$ : the cosets in $\mathbf{M}$ of the subgroups $L_{\bar{b}}:=\left\{\bar{b}^{T} \bar{a} \mid \bar{a} \in \mathbb{F}_{q}^{t}\right\}$, for all $\bar{b} \in$ $\mathbb{F}_{q}^{n+1} \backslash\{\overline{0}\}$.

There are exactly $\frac{q^{n}-1}{q-1}$ lines of type $L_{\bar{b}}$, since $L_{\bar{b}}=L_{\bar{c}}$, when $\bar{b}$ and $\bar{c}$ are scalar multiples of each other.

Note that this also provides a nice description of the point graph of our geometry as a Cayley graph: the vertices are the elements of $\mathbf{M}$, and two vertices are adjacent if and only if their difference (in $\mathbf{M}$ ) is of the form $\bar{b}^{T} \bar{a}$ for some $\bar{a} \in \mathbb{F}_{q}^{t}$ and some $\bar{b} \in \mathbb{F}_{q}^{n+1} \backslash\{\overline{0}\}$. 
Next we will find the lowest dimensional affine space in which $X(n, t, q)$ naturally embeds, and establish the isomorphism with $T_{n}^{*}(\mathcal{S})$ in an algebraic way. Let $f(x)=m_{0}+m_{1} x+\cdots+m_{t-1} x^{t-1}+x^{t}$ be an irreducible monic polynomial of degree $t$ over $\mathbb{F}_{q}$ used to construct $\mathbb{F}_{q^{t}}$. Let $M$ be the companion matrix of $f(x)$, that is,

$$
M=\left(\begin{array}{cc}
0 & I_{t-1} \\
-m_{0} & -\bar{m}
\end{array}\right)
$$

with $\bar{m}=\left(m_{1}, \ldots, m_{t-1}\right)$. Then it is well known from linear algebra (see for example [15], Theorem 3.3.14) that $f(x)$ is the minimal polynomial of $M$. Consequently, if we define

$$
H=\left\{a_{0} I_{t}+a_{1} M+\cdots+a_{t-1} M^{t-1} \mid a_{i} \in \mathbb{F}_{q}\right\},
$$

then $H$ has the structure of $\mathbb{F}_{q^{t}}$ under usual matrix addition and multiplication (this construction for example also appears in [18]). It follows that $H \backslash\{0\}$ acts sharply transitively on the points of $\operatorname{AG}(t, q)$ different from $(0, \ldots, 0)$.

Now define an action of $H$ on $\mathbf{M}$ in the following way:

$$
(H, \mathbf{M}) \rightarrow \mathbf{M}:\left(C, A_{P}\right) \mapsto A_{P} C .
$$

It is readily checked that this makes $\mathbf{M}$ into an $H$-vector space, that is,

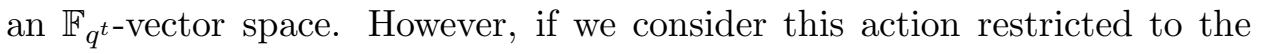
subgroups $L_{\bar{b}}$ of $\mathbf{M}$, then we see that we in fact obtain an action

$$
\left(H, L_{\bar{b}}\right) \rightarrow L_{\bar{b}}
$$

This makes the $L_{\bar{b}}$ into $H$-vector subspaces of the $H$-vector space $\mathbf{M}$.

From the above it now follows that we can view our geometry $X(n, t, q)$ as a geometry embedded in an $(n+1)$-dimensional vector space over $\mathbb{F}_{q^{t}}$, where the lines through $I$ correspond to certain vector subspaces and the other lines to cosets of these subspaces, which are parallel subspaces when seen in $\operatorname{AG}\left(n, q^{t}\right)$. Since the lines of $X(n, t, q)$ have size $q^{t}=|H|=\left|\mathbb{F}_{q^{t}}\right|$, these subspaces are one-dimensional and we obtain a linear representation of $X(n, t, q)$ as a point set in $\mathrm{PG}\left(n, q^{t}\right)$.

Let $q=p^{h}, p$ prime. Define the following

$$
\mathcal{A}=\left\{(A, B, C, l) \mid A \in \mathbf{M}, B \in \mathrm{GL}(n+1, q), C \in \mathrm{GL}(t, q), l \in \mathbb{Z}_{h}\right\},
$$

and a binary operation $\circ$ on $\mathcal{A}$ as follows

$\left(A_{2}, B_{2}, C_{2}, l_{2}\right) \circ\left(A_{1}, B_{1}, C_{1}, l_{1}\right)=\left(B_{2}^{p^{-l_{1}}} A_{1} C_{2}^{p^{-l_{1}}}+A_{2}^{p^{-l_{1}}}, B_{2}^{p^{-l_{1}}} B_{1}, C_{1} C_{2}^{p^{-l_{1}}}, l_{1}+l_{2}\right)$.

Then $\mathcal{A}$, is easily checked to be a group. 
Next define an action of $\mathcal{A}$ on the points of $\mathcal{M}$ as follows

$$
\left((A, B, C, l), A_{P}\right) \mapsto\left(B A_{P} C+A\right)^{p^{l}} .
$$

A simple verification now shows that this makes $\mathcal{A}$ into a group of automorphisms of $\mathcal{M}$. The kernel of the described action clearly is $K=$ $\left\{\left(0, \lambda I_{n+1}, \lambda^{-1} I_{t}, 0\right) \mid \lambda \in \mathbb{F}_{q}^{*}\right\}$.

Elements of the form $\left(A, I_{n+1}, I_{t}, 0\right)$ map every element of $\mathcal{L}_{\mathcal{M}}$ to one of its cosets, meaning it fixes the point set at infinity of the linear representation while permuting the lines that go through a given point at infinity. An element $\left(0, I_{n+1}, C, 0\right)$ fixes every subgroup $L_{\bar{b}}$ and permutes the group elements of every subgroup, meaning it fixes the point set at infinity and the affine point corresponding to the zero matrix (meaning the space $I$ ), while permuting the points of every line of $\mathcal{P}$ through this point. Elements of the form $\left(0, I_{n+1}, I_{t}, l\right)$ provide the semi-linear maps corresponding to the elements of $\operatorname{Aut}\left(\mathbb{F}_{q}\right)$. The action of the subgroup $\left\{\left(0, B, I_{t}, 0\right) \mid B \in \mathrm{GL}(n+1, q)\right\} \leq \mathcal{A}$ fixes the point corresponding to the zero matrix (that is, the origin in the corresponding linear representation) while permuting the lines that go through it. Hence this subgroup stabilises the point set at infinity of the linear representation.

We still need to uncover this point set at infinity. With every vector $\bar{b} \in \mathbb{F}_{q}^{n+1} \backslash\{\overline{0}\}$, up to scalar multiple, there is a corresponding point at infinity. Define a point-line incidence structure with as points the subgroups $L_{\bar{b}}$ and as lines the set of subgroups $\left\{L_{\overline{b_{i}}} \mid i=0, \ldots, q\right\}$ where the vectors $\overline{b_{i}}$ lie in a plane, meaning, the corresponding projective points lie on a projective line. This means the lines through the origin of the corresponding linear representation correspond naturally to the structure $\operatorname{PG}(n, q)$. Since this structure is stabilised by the subgroup $\left\{\left(0, B, I_{t}, 0\right) \mid B \in \mathrm{GL}(n+1, q)\right\}$ of the automorphism group of $\mathcal{M}$, we know that our point set at infinity is a subgeometry isomorphic to $\mathrm{PG}(n, q)$ and hence that $\mathcal{M} \cong T_{n}^{*}(\mathcal{S})$.

Finally, since this group has the size of the full automorphism group

$$
\frac{|\mathcal{A}|}{|K|}=q^{(n+1) t}|\mathrm{GL}(n+1, q)||\mathrm{GL}(t, q)|\left|\operatorname{Aut}\left(\mathbb{F}_{q}\right)\right| /(q-1)=\left|\operatorname{P\Gamma L}(n+t+1, q)_{\pi}\right|,
$$

we see that $\mathcal{A}$ provides a natural description of the full automorphism group of $T_{n}^{*}(\mathcal{S})$.

From this section we arrive to the following conclusion.

Theorem 4.1. The geometries $X(n, t, q)$ and $T_{n}^{*}(\mathcal{S})$ are isomorphic and $\operatorname{Aut}\left(T_{n}^{*}(\mathcal{S})\right) \cong \operatorname{Aut}(X(n, t, q)) \cong \operatorname{P\Gamma L}(n+t+1, q)_{\pi}$. 


\section{The linear representation and its automorphism group}

In this section, by some easy counting, we will show that while the automorphism group of $T_{n}^{*}(\mathcal{S})$ is not induced by collineations of its ambient space, there is yet another setting where we do see that the automorphisms are induced by collineations of the ambient space, namely when we consider the generalised linear representation isomorphic to $T_{n}^{*}(\mathcal{S})$.

Definition. Let $\mathcal{K}$ be a set of disjoint $(t-1)$-dimensional subspaces in $\Pi_{\infty} \cong$ $\mathrm{PG}(m, q), q$ a prime power. Embed $\Pi_{\infty}$ as a hyperplane in $\mathrm{PG}(m+1, q)$. The generalised linear representation $T_{m, t-1}^{*}(\mathcal{K})$ of $\mathcal{K}$ is the incidence structure $\left(\mathcal{P}^{\prime}, \mathcal{L}^{\prime}\right)$ with natural incidence and with:

$\mathcal{P}^{\prime}$ : the affine points of $\mathrm{PG}(m+1, q)$, i.e. the points of $\mathrm{PG}(m+1, q) \backslash \Pi_{\infty}$,

$\mathcal{L}^{\prime}$ : the $t$-dimensional spaces of $\operatorname{PG}(m+1, q)$ containing a $(t-1)$-space of $\mathcal{K}$, but not lying in $\Pi_{\infty}$.

When $t=1$, clearly this definition coincides with the definition of a linear representation.

By field reduction, the points of $\mathrm{PG}\left(n, q^{t}\right)$ correspond to the elements of a Desarguesian $(t-1)$-spread $\mathcal{D}_{\infty}$ of $J_{\infty} \cong \mathrm{PG}(t(n+1)-1, q)$. We will denote the element of $\mathcal{D}_{\infty}$ corresponding to a point $P$ of $\mathrm{PG}\left(n, q^{t}\right)$ by $\mathcal{F}(P)$, and define $\mathcal{F}(\pi):=\{\mathcal{F}(P) \mid P \in \pi\}$ for a subset $\pi$ of $\mathrm{PG}\left(n, q^{t}\right)$.

If $\mathcal{S}$ is a subgeometry $\operatorname{PG}(n, q)$ of $H_{\infty}$, then the set $\mathcal{F}(\mathcal{S})$ is a set of $\frac{q^{n+1}-1}{q-1}$ disjoint $(t-1)$-spaces in $J_{\infty}$. It is well-known that this set forms one of the two systems of the Segre variety $S_{n, t-1}$ in $\operatorname{PG}(t(n+1)-1, q)$. For more information on field reduction and this Segre variety we refer to [17.

Let us now recall the representation of Barlotti-Cofman [3] of PG(n+ $\left.1, q^{t}\right)$ inside $\mathrm{PG}(t(n+1), q)$. Here, the points of the hyperplane $H_{\infty} \cong$ $\mathrm{PG}\left(n, q^{t}\right)$ in $\mathrm{PG}\left(n+1, q^{t}\right)$ are represented as $(t-1)$-dimensional spaces of a Desarguesian spread $\mathcal{D}_{\infty}$ in $J_{\infty} \cong \operatorname{PG}(t(n+1)-1, q)$. The affine points of $\mathrm{PG}\left(n+1, q^{t}\right)$ with respect to $H_{\infty}$ can be identified with the affine points of a projective space $\mathrm{PG}(t(n+1), q)$, with respect to the hyperplane $J_{\infty}$. The lines of $\mathrm{PG}\left(n+1, q^{t}\right)$ intersecting $H_{\infty}$ in a point correspond to the $t$-dimensional spaces of $\mathrm{PG}(t(n+1), q)$, meeting $J_{\infty}$ in an element of $\mathcal{D}_{\infty}$.

We consider the Barlotti-Cofman representation of the points and lines of the linear representation $T_{n}^{*}(\mathcal{S})$. This means that the points of $\mathcal{S}$ in the hyperplane $H_{\infty}$ are represented as the set of elements $\mathbb{F}(\mathcal{S})$ in the Desarguesian $(t-1)$-spread $\mathcal{D}_{\infty}$, the affine points of $\mathrm{PG}\left(n+1, q^{t}\right)$ correspond to affine points of $\mathrm{PG}(t(n+1), q)$ and the lines of $T_{n}^{*}(\mathcal{S})$ correspond to $t$-spaces, having an element of $\mathbb{F}(\mathcal{S})$ at infinity. In this way we get the generalised linear representation $T_{t(n+1)-1, t-1}^{*}(\mathcal{F}(\mathcal{S}))$, which is then clearly isomorphic to $T_{n}^{*}(\mathcal{S})$. 
It is clear that the group $\operatorname{P\Gamma L}(t(n+1)+1, q)_{\mathcal{F}(\mathcal{S})}$ stabilises the generalised linear representation $T_{t(n+1)-1, t-1}^{*}(\mathcal{F}(\mathcal{S}))$ and hence is isomorphic to a subgroup of $\operatorname{Aut}(X(n, t, q)) \cong \operatorname{P\Gamma L}(n+t+1, q)_{\pi}$. We will see by counting that the groups are in fact isomorphic.

Definition. For a hyperplane $H$ of $\mathrm{PG}(m, q)$ we denote the set of elements of $\operatorname{P\Gamma L}(m+1, q)$ fixing all points of the hyperplane $H$ as $\operatorname{Persp}_{q}(H)$. Note that this is in fact a subgroup of $\operatorname{PGL}(m+1, q)$. Clearly, the group $\operatorname{Persp}_{q}(H)$ has size $\left|\operatorname{Persp}_{q}(H)\right|=q^{m}(q-1)$.

Result 5.1. 6] Suppose $\mathcal{K}$ is a subset of a hyperplane $H$ of $\mathrm{PG}(m, q)$ such that $\langle\mathcal{K}\rangle=H$. The group $\mathrm{P} \Gamma \mathrm{L}(m+1, q)_{\mathcal{K}}$ is an extension of $\operatorname{Persp}_{q}(H)$ by $\operatorname{P\Gamma L}(m, q)_{\mathcal{K}}$ and $\operatorname{PGL}(m+1, q)_{\mathcal{K}}$ is an extension of $\operatorname{Persp}_{q}(H)$ by $\operatorname{PGL}(m, q)_{\mathcal{K}}$.

Result 5.2. [14, Theorem 25.5.13] The projective automorphism group of a Segre variety $S_{l, k}$ of $\mathrm{PG}((l+1)(k+1)-1, q)$ is either isomorphic to $\operatorname{PGL}(l+1, q) \times \operatorname{PGL}(k+1, q)$ if $l \neq k$ or is isomorphic to $(\operatorname{PGL}(l+1, q) \times$ $\operatorname{PGL}(k+1, q)) \rtimes C_{2}$ if $l=k$.

To denote the number of $(k-1)$-dimensional subspaces of $\mathrm{PG}(m-1, q)$, we will use the following notation:

$$
\left[\begin{array}{c}
m \\
k
\end{array}\right]_{q}=\frac{\left(q^{m}-1\right)\left(q^{m-1}-1\right) \ldots\left(q^{m-k+1}-1\right)}{\left(q^{k}-1\right)\left(q^{k-1}-1\right) \ldots(q-1)} .
$$

Theorem 5.3. $\operatorname{Aut}\left(T_{n}^{*}(\mathcal{S})\right) \cong \operatorname{P\Gamma L}(n+t+1, q)_{\pi} \cong \operatorname{P\Gamma L}(t(n+1)+1, q)_{\mathcal{F}(\mathcal{S})}$

Proof. The full automorphism group of $T_{n}^{*}(\mathcal{S})$ is isomorphic to $\mathrm{P} \Gamma \mathrm{L}(n+t+$ $1, q)_{\pi}$, see Theorem 4.1. Since $\operatorname{P\Gamma L}(n+t+1, q)$ acts transitively on the $n$-spaces of $\mathrm{PG}(n+t, q)$, we find the following:

$$
\begin{aligned}
\left|\mathrm{P} \Gamma \mathrm{L}(n+t+1, q)_{\pi}\right| & =\frac{|\mathrm{P} \Gamma \mathrm{L}(n+t+1, q)|}{\left[\begin{array}{c}
n+t+1 \\
n+1
\end{array}\right]_{q}} \\
& =q^{t(n+1)} q^{\frac{t(t-1)}{2}}\left(q^{t}-1\right) \ldots(q-1)|\mathrm{P} \Gamma \mathrm{L}(n+1, q)| .
\end{aligned}
$$

Now we calculate the size of $\operatorname{P\Gamma L}(t(n+1)+1, q)_{\mathcal{F}(\mathcal{S})}$. By Result 5.1, since $\mathcal{F}(\mathcal{S})$ spans $J_{\infty}$, we find:

$$
\left|\operatorname{P\Gamma L}(t(n+1)+1, q)_{\mathcal{F}(\mathcal{S})}\right|=\left|\operatorname{Persp}_{q}\left(J_{\infty}\right)\right|\left|\operatorname{P\Gamma L}(t(n+1), q)_{\mathcal{F}(\mathcal{S})}\right| .
$$

As seen before, the set of points contained in $\mathcal{F}(\mathcal{S})$ forms a Segre variety $S_{n, t-1}$. Hence the stabiliser of $\mathcal{F}(\mathcal{S})$ is the stabiliser of the Segre variety that in the case $t=n+1$ does not switch the two systems. Thus, by Result 5.2 we find $\operatorname{PGL}(t(n+1), q)_{\mathcal{F}(\mathcal{S})}=\operatorname{PGL}(n+1, q) \times \operatorname{PGL}(t, q)$. The semilinear 
automorphisms stabilising any one of the systems of the Segre variety naturally extend to elements of $\operatorname{P\Gamma L}(t(n+1), q)$. Hence $\left|\operatorname{P\Gamma L}(t(n+1), q)_{\mathcal{F}(\mathcal{S})}\right|=$ $|\operatorname{PGL}(n+1, q)||\operatorname{PGL}(t, q)|\left|\operatorname{Aut}\left(\mathbb{F}_{q}\right)\right|$.

We conclude that $\operatorname{P\Gamma L}(t(n+1)+1, q)_{\mathcal{F}(\mathcal{S})}$ has the same size as $\operatorname{P\Gamma L}(n+$ $t+1, q)_{\pi}$ :

$$
\begin{aligned}
\left|\operatorname{P\Gamma L}(t(n+1)+1, q)_{\mathcal{F}(\mathcal{S})}\right| & =\left|\operatorname{Persp}_{q}\left(J_{\infty}\right)\right||\operatorname{PGL}(n+1, q)||\operatorname{PGL}(t, q)|\left|\operatorname{Aut}\left(\mathbb{F}_{q}\right)\right| \\
& =\left|\operatorname{Persp}_{q}\left(J_{\infty}\right)\right||\operatorname{P\Gamma L}(n+1, q)||\operatorname{PGL}(t, q)| \\
& =q^{t(n+1)}(q-1) q^{\frac{t(t-1)}{2}}\left(q^{t}-1\right) \ldots\left(q^{2}-1\right)|\operatorname{P\Gamma L}(n+1, q)| .
\end{aligned}
$$

Clearly every collineation of $\operatorname{P\Gamma L}(t(n+1)+1, q)_{\mathcal{F}(\mathcal{S})}$ is a non-trivial element of $\operatorname{Aut}\left(T_{n}^{*}(\mathcal{S})\right)$. Both groups have the same size, so $\operatorname{Aut}\left(T_{n}^{*}(\mathcal{S})\right) \cong$ $\operatorname{P\Gamma L}(t(n+1)+1, q)_{\mathcal{F}(\mathcal{S})}$.

The subgroup of the automorphism group of the linear representation $T_{n}^{*}(\mathcal{S})$ for which the elements are induced by collineations of the space $\mathrm{PG}\left(n+1, q^{t}\right)$ will be called the geometric automorphism group. Since $\mathcal{S}$ spans $H_{\infty}$, this group is isomorphic to $\operatorname{P\Gamma L}\left(n+2, q^{t}\right)_{\mathcal{S}}$.

Theorem 5.4. The full automorphism group of $T_{n}^{*}(\mathcal{S})$ is $\frac{1}{t} q^{\frac{t(t-1)}{2}}\left(q^{t-1}-\right.$ $1) \ldots\left(q^{2}-1\right)(q-1)$ times larger than the geometric automorphism group of $T_{n}^{*}(\mathcal{S})$.

Proof. The geometric automorphism group of $T_{n}^{*}(\mathcal{S})$ is isomorphic to $\mathrm{P} \Gamma \mathrm{L}(n+$ $\left.2, q^{t}\right)_{\mathcal{S}}$ and has the following size:

$$
\begin{aligned}
\left|\operatorname{P\Gamma L}\left(n+2, q^{t}\right)_{\mathcal{S}}\right| & =\left|\operatorname{Persp}_{q^{t}}\left(H_{\infty}\right)\right|\left|\operatorname{Aut}\left(\mathbb{F}_{q^{t}}\right) / \operatorname{Aut}\left(\mathbb{F}_{q}\right)\right||\operatorname{P\Gamma L}(n+1, q)| \\
& =q^{t(n+1)}\left(q^{t}-1\right) t|\operatorname{P\Gamma L}(n+1, q)| .
\end{aligned}
$$

We end with a full answer to the isomorphism problem of linear representations $T_{n}^{*}(\mathcal{K})$ and $T_{n}^{*}\left(\mathcal{K}^{\prime}\right)$ of point sets $\mathcal{K}, \mathcal{K}^{\prime}$.

Theorem 5.5. Let $\mathcal{K}$ and $\mathcal{K}^{\prime}$ denote point sets in $H_{\infty} \cong \mathrm{PG}\left(n, q^{t}\right)$ such that the closure $\overline{\mathcal{K}}$ is a subgeometry $\mathrm{PG}(n, q)$ of $H_{\infty}$ and let $\alpha$ be an isomorphism between $T_{n}^{*}(\mathcal{K})$ and $T_{n}^{*}\left(\mathcal{K}^{\prime}\right)$. Then $\alpha$ is induced by an element of $\mathrm{P} \Gamma \mathrm{L}(t(n+$ $1)+1, q)_{J_{\infty}}$ mapping $\mathcal{F}(\mathcal{K})$ onto $\mathcal{F}\left(\mathcal{K}^{\prime}\right)$.

\section{References}

[1] R.W. Ahrens and G. Szekeres. On a combinatorial generalization of 27 lines associated with a cubic surface. J. Austral. Math. Soc. 10 (1969), $485-492$.

[2] L. Bader and G. Lunardon. Desarguesian spreads. Ricerche mat. 60 (2011), 15-37. 
[3] A. Barlotti and J. Cofman. Finite Sperner spaces constructed from projective and affine spaces. Abh. Math. Sem. Univ. Hamburg 40 (1974), $231-241$.

[4] A. Bichara, F. Mazzocca and C. Somma. On the classification of generalized quadrangles in a finite affine space $\operatorname{AG}\left(3,2^{h}\right)$. Boll. Un. Mat. Ital. B (5) 17 (1) (1980), 298-307.

[5] R.H. Bruck, R.H. Finite nets. I. Numerical invariants. Can. J. Math 3 (1951), 94-107.

[6] P. Cara, S. Rottey and G. Van de Voorde. The isomorphism problem for linear representations and their graphs. To appear in Adv. Geom.

[7] I. Debroey. Semi-partiële meetkunden. PhD Thesis. Ghent University, 1978.

[8] F. De Clerck. Een kombinatorische studie van de eindige partiele meetkunden. PhD Thesis. Ghent University, 1978.

[9] S. De Winter. Elation and translation semipartial geometries. J. Combin. Theory Ser. A 108 (2004), 313-330.

[10] S. De Winter. Non-isomorphic semipartial geometries. Des. Codes Cryptogr. 47 (2008), 3-9.

[11] T. Grundhoefer, M. Joswig and M. Stroppel. Slanted symplectic quadrangles. Geom. Dedicata 49 (1994), 143-154.

[12] M. Hall, Jr. Affine generalized quadrilaterals. Studies in Pure Mathematics. Academia Press, London (1971), 113-116.

[13] M. Hall, Jr. The Theory of Groups. AMS Chelsea Publishing, 1999.

[14] J.W.P. Hirschfeld and J.A. Thas. General Galois Geometries. Oxford University Press, Oxford, 1991.

[15] R.A. Horn and C.R. Johnson. Matrix analysis. Cambridge University Press, Cambridge, 1985.

[16] D. Hughes and F. Piper. Projective Planes. Springer-Verlag, 1973.

[17] M. Lavrauw and G. Van de Voorde. Field reduction and linear sets in finite geometry. To appear in AMS Contemp. Math, American. Math Soc.. arxiv.org/abs/1310.8522.

[18] R. Lidl and H. Niederreiter. Finite Fields. Encyclopedia of Mathematics and its Applications 20, Cambridge University Press, 1997. 
[19] S.E. Payne and J.A. Thas. Finite Generalized Quadrangles. Research Notes in Mathematics 110, Pitman, Boston, 1984.

Affiliation of the authors:

sgdewint@mtu.edu

Department of Mathematics, Michigan Technological University

1400 Townsend Drive, Houghton, MI 49931, United States

srottey@vub.ac.be

Department of Mathematics, Vrije Universiteit Brussel

Pleinlaan 2, 1050 Brussel, Belgium

gvdevoorde@cage.ugent.be

Department of Mathematics, UGent

Krijgslaan 281-S22, 9000 Gent, Belgium 